

А. Я. Мальцев, Нелокальные скобки Пуассона и метод Уизема, УМН, 1999, том 54, выпуск 6, 167-168

DOI: https://doi.org/10.4213/rm241

Использование Общероссийского математического портала Math-Net.Ru подразумевает, что вы прочитали и согласны с пользовательским соглашением

http://www.mathnet.ru/rus/agreement

Параметры загрузки:

IP : 54.198 .64 .247

26 апреля 2023 г., 03:56:44 


\title{
НЕЛОКАЛЬНЫЕ СКОБКИ ПУАССОНА И МЕТОД УИЗЕМА
}

\author{
А. Я. МАЛЬЦЕВ
}

Мы рассматриваем нелокальные теоретико-полевые скобки Пуассона на пространстве функций $\varphi(x)=\left(\varphi^{1}(x), \ldots, \varphi^{n}(x)\right)$ вида:

$$
\begin{aligned}
\left\{\varphi^{i}(x), \varphi^{j}(y)\right\}=\sum_{k \geqslant 0} B_{k}^{i j}\left(\varphi, \varphi_{x}, \ldots\right) & \delta^{(k)}(x-y) \\
& +\sum_{k \geqslant 0} e_{k} S_{(k)}^{i}\left(\varphi, \varphi_{x}, \ldots\right) \nu(x-y) S_{(k)}^{j}\left(\varphi, \varphi_{y}, \ldots\right),
\end{aligned}
$$

где $e_{k}= \pm 1, \nu(x-y)=-\nu(y-x), \partial_{x} \nu(x-y)=\delta(x-y)$ и обе суммы содержат конечное число членов, зависящих от конечного числа производных $\varphi$ по $x$. Как хорошо известно, скобки (1) обыкновенно встречаются в теории "интегрируемых" систем типа Кортевега-де Фриза, Нелинейного Шрёдингера и т. п.

Е.В. Ферапонтовым ([1], см. также [2]) был введен важный класс скобок (1), обладающий замечательными дифференциально-геометрическими свойствами, вытекающими из тождества Якоби:

(2) $\quad\left\{U^{\nu}(X), U^{\mu}(Y)\right\}=g^{\nu \mu}(U) \delta^{\prime}(X-Y)+b_{\lambda}^{\nu \mu}(U) U_{X}^{\lambda} \delta(X-Y)$

$$
+\sum_{k \geqslant 0} e_{k} S_{(k) \lambda}^{\nu}(U) U_{X}^{\lambda} \nu(X-Y) S_{(k) \delta}^{\mu}(U) U_{Y}^{\delta}, \quad 1 \leqslant \nu, \mu, \lambda, \delta \leqslant N .
$$

ТЕорема 1. Для любого гамильтонова оператора, записанного в форме (1), потоки $\dot{\varphi}^{i}=S_{(k)}^{i}\left(\varphi, \varphi_{x}, \ldots\right)$ коммутируют друг с другом и сохраняют гамильтонову структу$p y(1)$.

Заметим здесь, что первое утверждение теоремы для нелокальных скобок Ферапонтова (2) было ранее доказано Е. В. Ферапонтовым из дифференциально-геометрических соображений (см. библиограффию в [2]). В [2] может быть найдена также полная классификация скобок (2) с дифференциально-геометрической точки зрения.

Пусть теперь мы имеем локальную гамильтонову систему

$$
\varphi_{t}^{i}=Q^{i}\left(\varphi, \varphi_{x}, \ldots\right)
$$

порожденную локальным функционалом $H=\int \mathscr{P}_{H}\left(\varphi, \varphi_{x}, \ldots\right) d x$.

Метод Уизема ([3]) дает нам эволюционные уравнения на медленно промодулированные параметры $m$-фозных решений (3) в форме:

$$
U_{T}^{\nu}=V_{\mu}^{\nu}(U) U_{X}^{\mu}, \quad \nu, \mu=1, \ldots, N,
$$

где $N$ - полное число параметров $m$-фазных решений исключая начальные фазы. При некоторых условиях "регулярности" (см. [4]) мы можем предложить процедуру усреднения скобки (1) и получения скобки Ферапонтова для системы Уизема (4).

Теорема 2. Пусть имеется скобка Пуассона (1) и система (3), порожденная локальным гамильтонианом $H$ и обладающая $N$-параметрическим $(N \geqslant 2 m)$ полным семейством $m$-фазных решений, не считая $m$ начальных фазовых сдвигов $\theta_{0}^{\alpha}$. Пусть система (3) имеет также $N$ коммутативных локальных трансляционно-инвариантных интегралов

$$
I^{\nu}=\int \mathscr{P}^{\nu}\left(\varphi, \varphi_{x}, \ldots\right) d x, \quad\left\{I^{\nu}, H\right\}=0, \quad\left\{I^{\nu}, I^{\mu}\right\}=0,
$$

порождающих локальные потоки в силу скобки Пуассона (1) и таких, что усредненные плотности $\left\langle\mathscr{P}^{\nu}\right\rangle$ могут быть взяты за параметры $U^{\nu}$ на пространстве т-фазных решений (3). 
Вычислим попарные скобки Пуассона $\mathscr{P}^{\nu}\left(\varphi, \varphi_{x}, \ldots\right)$ в форме

$$
\begin{aligned}
\left\{\mathscr{P}^{\nu}\left(\varphi, \varphi_{x}, \ldots\right), \mathscr{P}^{\mu}\left(\varphi, \varphi_{y}, \ldots\right)\right\} & =\sum_{k \geqslant 0} A_{k}^{\nu \mu}\left(\varphi, \varphi_{x}, \ldots\right) \delta^{(k)}(x-y) \\
& +\sum_{k \geqslant 0} e_{k}\left(F_{(k)}^{\nu}\left(\varphi, \varphi_{x}, \ldots\right)\right)_{x} \nu(x-y)\left(F_{(k)}^{\mu}\left(\varphi, \varphi_{y}, \ldots\right)\right)_{y}
\end{aligned}
$$

(обе суммы содержат конечное число членов). Мы имеем здесь полные производнье функций $F_{(k)}^{\nu}$ и $F_{(k)}^{\mu}$ по х и у как следствие того, что функциональ $I^{\nu}$ и $I^{\mu}$ порожсдают локальные потоки в силу (1). Из коммутативности набора $\left\{I^{\nu}\right\}$ мы получаем также $A_{0}^{\nu \mu}\left(\varphi, \varphi_{x}, \ldots\right)+\sum_{k \geqslant 0} e_{k}\left(F_{(k)}^{\nu}\left(\varphi, \varphi_{x}, \ldots\right)\right)_{x} F_{(k)}^{\mu}\left(\varphi, \varphi_{x}, \ldots\right) \equiv \partial_{x} Q^{\nu \mu}\left(\varphi, \varphi_{x}, \ldots\right) \partial_{\Omega s}$ некоторьх функиий $Q^{\nu \mu}\left(\varphi, \varphi_{x}, \ldots\right)$.

Тогда для “медленньх" координат $U^{\nu}(X)=\left\langle\mathscr{P}^{\nu}\right\rangle(X)$ мъ можем определить скобку Пуассона согласно формуле:

$$
\begin{aligned}
\left\{(5)\left\{U^{\nu}(X), U^{\mu}(Y)\right\}=[\right. & \left.\left\langle A_{1}^{\nu \mu}\right\rangle(X)+\sum_{k \geqslant 0} e_{k}\left(\left\langle F_{(k)}^{\nu} F_{(k)}^{\mu}\right\rangle-\left\langle F_{(k)}^{\nu}\right\rangle\left\langle F_{(k)}^{\mu}\right\rangle\right)(X)\right] \delta^{\prime}(X-Y) \\
& +\left[\frac{\partial\left\langle Q^{\nu \mu}\right\rangle(X)}{\partial X}-\sum_{k \geqslant 0} e_{k} \frac{\partial\left\langle F_{(k)}^{\nu}\right\rangle(X)}{\partial X}\left\langle F_{(k)}^{\mu}\right\rangle(X)\right] \delta(X-Y) \\
& +\sum_{k \geqslant 0} e_{k} \frac{\partial\left\langle F_{(k)}^{\nu}\right\rangle(X)}{\partial X} \nu(X-Y) \frac{\partial\left\langle F_{(k)}^{\mu}\right\rangle(Y)}{\partial Y},
\end{aligned}
$$

где $\langle\cdot\rangle$ означает усреднение на пространстве т-фазных решений (3) и все усредненные величинь являются функциями $U(X)$ и $U(Y)$ в соответствующих точках $X$ и $Y$. Скобка (5) удовлетворяет тождеству Якоби и является инвариантной по отношению к вьбору $\left\{I^{1}, \ldots, I^{N}\right\}$, т.е. если $U^{\nu}=\left\langle\mathscr{P}^{\nu}\right\rangle, \widetilde{U}^{\nu}=\left\langle\widetilde{\mathscr{P}}^{\nu}\right\rangle u\left\{U^{\nu}(X), U^{\mu}(Y)\right\}$, $\left\{\widetilde{U}^{\nu}(X), \widetilde{U}^{\mu}(Y)\right\}^{\prime}-$ скобки (5), построенные при помощи наборов $\left\{I^{\nu}\right\}$ u $\left\{\widetilde{I}^{\nu}\right\}$ соответственно, то:

$$
\left\{\widetilde{U}^{\nu}(X), \widetilde{U}^{\mu}(Y)\right\}^{\prime} \equiv \frac{\partial \widetilde{U}^{\nu}}{\partial U^{\lambda}}(X)\left\{U^{\lambda}(X), U^{\sigma}(Y)\right\} \frac{\partial \widetilde{U}^{\mu}}{\partial U^{\sigma}}(Y)
$$

Отметим здесь, что предложенная процедура является обобшением метода Дубровина-Новикова ([5]-[7]), введенного для локальных теоретико-полевых скобок.

ТеОрема 3. Гамильтониан $\bar{H}=\int\left\langle\mathscr{P}_{H}\right\rangle(U(X)) d X$ порожсдает в силу (5) локальныи поток, дающий уравнения Уизема для системы (3).

Подробное рассмотрение сформулированных результатов может быть найдено в [4].

Автор благодарен Б. А. Дубровину, С. П. Новикову, В. Л. Алексееву, О. И. Мохову, М. В. Павлову и Е. В. Ферапонтову за плодотворные обсуждения.

Автор также благодарит INTAS (грант № 96-0770) и РФФИ (грант № 97-01-00281) за частичную финансовую поддержку.

\section{СПИСОК ЛИТЕРАТУРЫ}

[1] Ferapontov E. V. // Amer. Math. Soc. Transl. (2). 1995. V. 170. P. 33-58. [2] Moхов О.И., Ферапонтов Е. В. // УМН. 1990. Т. 45. № 3. С. 191-192. [3] Уизем Дж. Линейные и нелинейные волны. М.: Мир, 1977. [4] Maltsev А. Ya. // solv-int/9910011. [5] Дубровин Б.А., Новиков С. П. // Докл. АН СССР. 1983. Т. 270. №4. С. 781-785. [6] Дубровин Б. А., Новиков С. П. // УМН. 1989. Т. 44. №6. С. 29-98. [7] Dubrovin В. А., Novikov S. P. // Sov. Sci. Rev. C. Math. Phys. 1993. V. 9. № 4. P. 1-136. 